\title{
Systematic review and meta-analysis of the effect of continuous cerebrospinal fluid drainage on keyhole surgery during the perioperative period
}

\author{
Jinzhou Feng ${ }^{1,2 \#}$, Qidong Liu ${ }^{1,2 \#}$, Deqian Cao ${ }^{1,2}$, Fajian Liu ${ }^{1,2}$ \\ ${ }^{1}$ Department of Neurosurgery, Sichuan Provincial People's Hospital, University of Electronic Science and Technology of China, Chengdu, China; \\ ${ }^{2}$ Chinese Academy of Sciences Sichuan Translational Medicine Research Hospital, Chengdu, China \\ Contributions: (I) Conception and design: J Feng, Q Liu; (II) Administrative support: Q Liu; (III) Provision of study materials or patients: D Cao; (IV) \\ Collection and assembly of data: All authors; (V) Data analysis and interpretation: J Feng, Q Liu, F Liu; (VI) Manuscript writing: All authors; (VII) \\ Final approval of the manuscript: All authors. \\ \#These authors contributed equally to this work. \\ Correspondence to: Deqian Cao; Fajian Liu. Department of Neurosurgery, Sichuan Provincial People's Hospital, University of Electronic Science and \\ Technology of China, Chengdu 610072, China; Chinese Academy of Sciences Sichuan Translational Medicine Research Hospital, Chengdu 610072, \\ China. Email: caodeqiansyy001@163.com; 1liufajianscpph@163.com.
}

Backgroundk Lumbar continuous drainage of fluid (LCDF) has become more widely used in the diagnosis and treatment of neurological diseases in recent years. The use of LCDF can enable a better understanding of the patient's condition and reduce the incidence of related complications. LCDF can also affect complications of perforation surgery, including mortality during hospitalization, cerebral vasospasm (CVS), bleeding, and aneurysmal subarachnoid hemorrhage (aSAH).

Methods: Articles published from library construction to April 2021 were searched for in the Englishlanguage databases PubMed, Cochrane Library, and Embase. All randomized controlled trials (RCTs) with LCDF and hole locking surgery were meta-analyzed using the Cochrane Collaboration's RevMan 5.3 software.

Results: Ten RCTs involving 1,092 patients (continuous drainage group, $\mathrm{n}=585$; control group, $\mathrm{n}=507$ ) were included in the meta-analysis. For the statistical different in incidence of perioperative cerebral infarction in the two groups, the odds ratio (OR) was 5.42 [95\% confidence interval (CI): $(2.71,10.83)$; $\mathrm{P}<0.00001]$, and for the statistical difference in the incidence of cerebral hemorrhage, the OR was 4.76 [95\% CI: (2.11, 10.76); $\mathrm{P}=0.0002]$. Perioperative complications were fewer in the LCDF-treated drainage group than in the conventional group.

Discussion: This meta-analysis of 10 RCTs confirmed that LCDF compared with other treatments is associated with a lower incidence of perioperative complications, such as cerebral hemorrhage, hydrocephalus, and cerebral infarction, as well as increased Glasgow Outcome Scale (GOS).

Keywords: Continuous lumbar large-pool cerebrospinal fluid drainage (continuous lumbar large-pool CSF drainage); locking surgery; neurosurgery; perioperative period; mate analysis

Submitted Aug 17, 2021. Accepted for publication Oct 20, 2021.

doi: 10.21037/apm-21-2728

View this article at: https://dx.doi.org/10.21037/apm-21-2728 


\section{Introduction}

Lumbar continuous drainage of fluid (LCDF) is a mature clinical technology used in the diagnosis and treatment of neurological diseases. The many advantages of LCDF include its simplicity, economy, and minimal trauma (1). This technique was first used to treat or prevent cerebrospinal fluid (CSF) leakage after craniocerebral and spinal surgery in patients at risk for CSF leakage after surgery (2). However, Ringel et al. (3) reported that while continuous CSF drainage could induce dural decompression and facilitate frontal lobe operation, it was associated with the occurrence of serious complications, including CSF leakage and meningitis, after surgery. Aneurysmal subarachnoid hemorrhage (aSAH) is a common cerebrovascular disease. Cerebral vasospasm (CVS) and rebleeding are all serious complications in the early stage after aSAH and can cause high mortality. In their study of 211 patients, Bakhshi et al. (4) observed the adverse effects of LCDF, and found iatrogenic spinal fluid leakage and Jiaxing meningocele to be the most common complications. Pathogens of meningitis include E. coli and Pseudomonas aeruginosa. Although lumbar large pool drainage technology has gradually matured, excessive drainage can lead to epidural hematoma and even brain hernia, retrograde infection, and symptoms of nerve root stimulation at the puncture site. Foreign scholars have explored methods to prevent complications of LCDF, which have included connecting an intravenous infusion pump to an external device to achieve a continuous and controlled flow rate (5). Although LCDF is a mature technology, its relationship with complications of locking surgery is still a topic of concern.

Keyhole surgery is an important minimally invasive neurosurgical approach. Wilson first proposed this concept in 1971, stating that locking holes can meet the needs of microscopic neurosurgery and this method improves the traditional craniotomy and limits the size of the surgical approach (6). The approach is simple and is widely used to treat hypertension, cerebral hemorrhage, intracranial aneurysm, and other intracranial diseases. However, various complications can still occur during perforation surgery, including cerebral hemorrhage, cerebral infarction, hydrocephalus, and infection. Researchers have attempted to reduce the incidence of complications during locking prior to surgery via various means, among which persistent drainage of CSF has received broad attention (7).

The aim of this meta-analysis was to summarize randomized controlled trials (RCTs) studying the effects of continuous lumbar large pool CSF drainage on perioperative complications in neurology patients undergoing locking surgery. We screened and metaanalyzed 8 RCTs, and reviewed case comparison results from China and overseas. Our findings will provide a scientific basis and theoretical reference for the application of continuous lumbar large-pool CSF drainage in neurology patients. Compared with the previous literatures, this study not only included perioperative complications, but also analyzed the mortality, CVS, rebleeding, and aSAH, which can better study the therapeutic effect of continuous lumbar cisternal CSF drainage. We present the following article in accordance with the PRISMA reporting checklist (available at https://dx.doi.org/10.21037/apm-21-2728).

\section{Methods}

\section{Strategy for article retrieval}

The PubMed, Cochrane Library, and Embase databases were searched for relevant RCTs. Keywords were searched for related keywords, and Medical Subject Headings (MeSH terms) for continuous lumbar drainage of fluid, keyhole surgery, and perioperative following the Cochrane Handbook for Systematic Reviews of Interventions (version 5.1.0) combined with subject search bar to "subarachnoid hemorrhage, lumbar drain". The search was restricted to RCTs published up to April 2021, and the start date was selected to overlap with the last systematic review of the prevention strategy, literature was searched using the following descriptive terms, such as LCDF, CVS, aSAH, infection, bleeding, history of chronic disease, paralysis, infection, hypotension, hyponatremia, smoking, small vessel disease infarction, risk factors for death, cerebrovascular disease, cerebral infarction, and the descriptive term of stroke. According to pre-formulated inclusion and exclusion criteria, obtain the full text of the literature included in the study.

\section{Inclusion and exclusion criteria}

The inclusion criteria were as follows: (I) RCTs, with either hidden or blind allocation; (II) study participants over 18 years old; (III) study participants undergoing neurosurgical perforation procedures for hydrocephalus, subarachnoid hemorrhage, intracranial infection, or rupture of aneurysm; (IV) intervention measures: control 
group treatment included routine treatment to restore the automatic cerebrovascular regulation mechanism, maintain effective blood volume and effective cerebral perfusion, control intracranial pressure, and prevent cerebral edema, and the drainage group underwent continuous drainage with a lumbar puncture; (V) main outcome indexes included hospitalization mortality, and the incidence of CVS, hemorrhage, and hydrocephalus; (VI) secondary outcome indexes included short-term clinical efficacy, 6-month prognosis, and adverse reactions.

The exclusion criteria were as follows: (I) studies with unclear data; (II) studies in Chinese; (III) does not contain the required disease data; (IV) non-controlled studies or non-RCTs; (V) studies with unreliable results; (VI) studies involving intermittent lumbar puncture discharge of spinal fluid or lateral ventricular flushing.

\section{Literature extraction}

Two independent researchers independently extracted data from the eligible articles. Differences which arose during data extraction were resolved through discussion or the opinion of a third researcher was sought. Data extracted from the articles included basic information (document topic, first author, author year, author information, and literature source), basic characteristics of the study participants (sex, age, study sample size, and baseline comparability), literature research methods, research scheme design, intervention measures in the experimental and control groups, outcome evaluation indicators, and outcome data.

\section{Quality assessment}

To determine the quality of the articles, a quality evaluation was conducted according to the bias risk assessment recommended by the RevMan (version 5.3). The evaluation considered the following seven elements, such as sequence generation and allocation concealment (selection bias or allocation bias), blinding of participants and personnel (performance bias), blinding of outcome assessors (detection bias), etc. Regarding these seven items, "Yes" meant that an article had a small risk of bias, and "No" meant that an article had a high degree of bias. If the study had not been fully reported in detail, the risk was unknown. The modified Jadad scale review was used to evaluate the quality of the attached research and literature. The evaluation included random sequence generation, allocation concealment, blinding, and tracking/exit; a score of 1 to 3 points indicated low quality, and a score of 4 to 7 points indicated high quality.

\section{Data extraction}

The results of the mate-analysis were expressed in forest maps, accompanied by the corresponding confidence intervals (CIs). If there was no overlap between the CIs of the individual results, statistical inhomogeneity was considered to exist between the studies. Combined stochastic and fixed models with acceptable inhomogeneity required further subgroup analysis. Studies were divided into subgroups according to design. When the inhomogeneity between different studies could not be ignored, the effect size for each subgroup was ignored to cope with inhomogeneity, and a combined statistical model was selected. A sensitivity analysis of the results was conducted to investigate whether the overall combined results were being influenced by a single study. Research of this paper can impact the mateanalysis results in this way. First, if a study is deleted, the constructive value of the size of the integrated composite effect is other than $95 \%$ of the integrated synthetic effect, and the results yield significantly different results.

\section{Sensitivity analysis}

Sensitivity analysis was used to evaluate whether the meta-analysis results were stable and reliable. Specifically, the change of meta-analysis results was observed by excluding some controversial studies, low-quality studies or analyzing the same group of data with different statistical methods/effect models. If the sensitivity analysis does not substantially change the results, the results are reliable. On the contrary, it should be careful in interpreting the results and drawing conclusions.

\section{Statistical analysis}

The Cochrane Collaboration's RevMan 5.3 software was used for the meta-analysis. The calculation method used the ratio [odds ratio (OR)] as the effect size with 95\% CI. The heterogeneity threshold for the included studies was $\alpha=0.1$. When no heterogeneity existed between the studies ( $\left.\mathrm{P}>0.1 ; \mathrm{I}^{2}<50 \%\right)$, the fixed-effects model was selected for meta-analysis; when heterogeneity existed $\left(\mathrm{I}^{2}>50 \%\right)$, the stochastic effect model was used for meta-analysis. $\mathrm{P}<0.05$ indicated a statistically significant difference. For single risk 


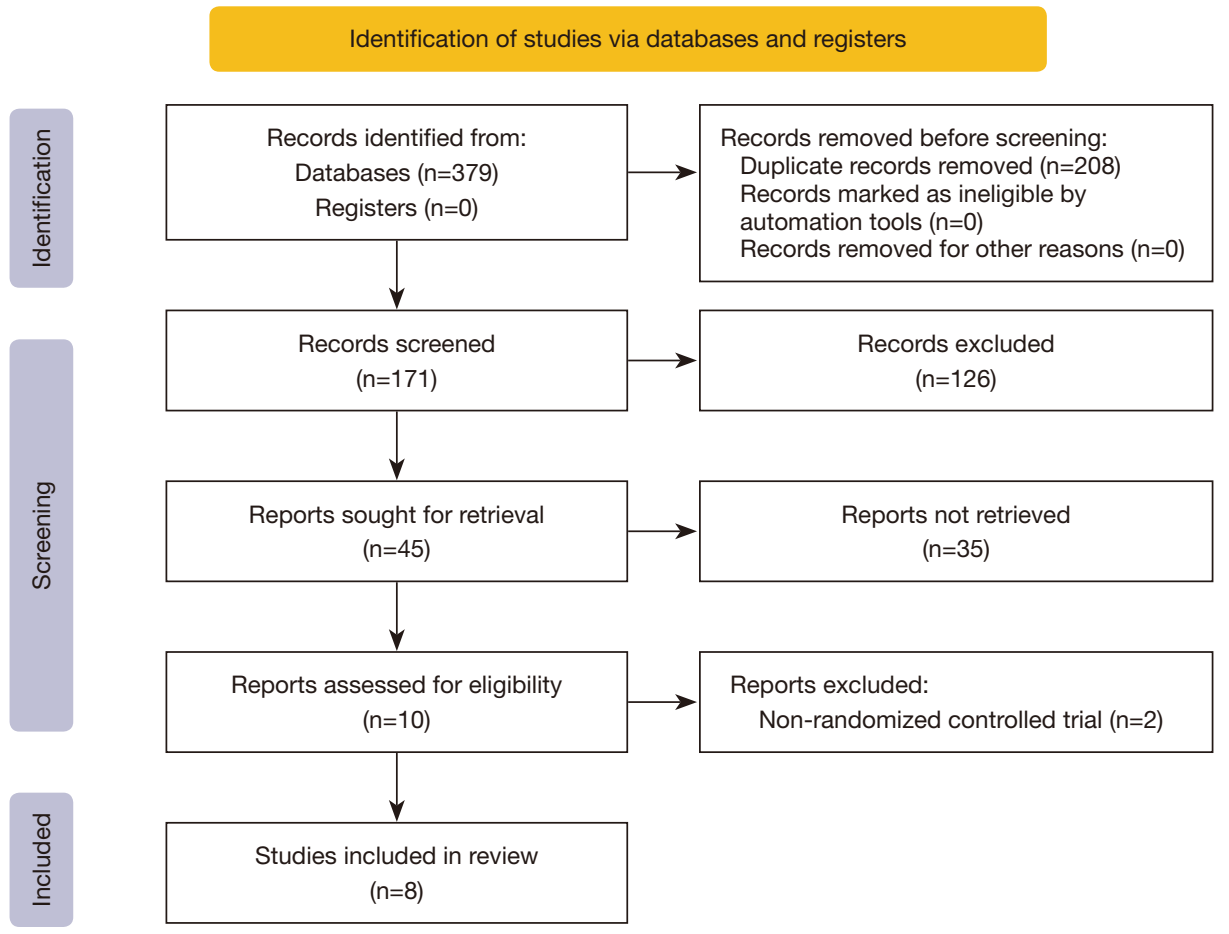

Figure 1 Flow chart for the article retrieval process.

factor analysis of more than 8 studies, funnel plots were used to analyze publication bias of risk factors.

\section{Results}

\section{Results of article retrieval}

A total of 379 relevant articles were retrieved, including 137 from PubMed, 129 from Embase, and 113 from OvidMedline. Of these articles, 208 duplicates were excluded, as were 126 articles that did not meet the inclusion criteria based on the reading of their text titles and abstracts. The full texts of the remaining articles were read, and 35 documents were subsequently excluded. Finally, 8 articles which met the inclusion criteria were included (8-15). The literature retrieval process is shown in Figure 1, and the basic information of the included articles is shown in Table 1.

\section{Bias risk assessment of the included articles}

The Cochrane Handbook (version 5.1) of the systematic review writing manual was adopted for assessment of the risk of bias in the 8 included articles. The results of the bias risk assessment are shown in Figures 2,3.

\section{Hydrocephalus}

Four articles reported the incidence of hydrocephalus during hospitalization. Hydrocephalus was experienced by 72 of 404 patients in the drainage group, and 24 of 315 patients in the conventional group. The results of heterogeneity analysis showed $\mathrm{I}^{2}=67 \%$ and $\mathrm{P}=0.03$, which indicated significant heterogeneity in the literature; consequently, a randomeffects model was used. The meta-analysis results showed $\mathrm{OR}=2.30,95 \% \mathrm{CI}:(0.90,5.89), \mathrm{Z}=1.74$, and $\mathrm{P}=0.08$; therefore, these results showed a no significant difference in the incidence of persistent drainage of CSF drainage between the drainage and conventional groups $(\mathrm{P}>0.05)$ (Figure 4). The funnel diagram was symmetrical (Figure 5), with most of the data corresponding to points within the $95 \%$ CI. This suggested that an incidence of hydrocephalus above $5 \mathrm{k}+10=95$ indicated that publication bias.

\section{Hematencephalon}

Eight studies reported the incidence of cerebral hemorrhage during hospitalization. In the analysis, 585 patients in the drainage group and 507 patients in the conventional group experienced cerebral hemorrhage. The results of heterogeneity analysis showed $\mathrm{I}^{2}=68 \%$ and $\mathrm{P}=0.003$, 
Table 1 Basic characteristics of the included articles

\begin{tabular}{|c|c|c|c|c|c|c|c|}
\hline Author & $\begin{array}{l}\text { Publication } \\
\text { year }\end{array}$ & $\begin{array}{l}\text { Drainage } \\
\text { group } \\
\text { (case) }\end{array}$ & $\begin{array}{l}\text { Control } \\
\text { group } \\
\text { (case) }\end{array}$ & Ending indicator & $\begin{array}{l}\text { Stochastic } \\
\text { method }\end{array}$ & Age (years old) & $\begin{array}{l}\text { Follow-up } \\
\text { time (months) }\end{array}$ \\
\hline Park (10) & 2015 & 126 & 108 & $\begin{array}{l}\text { Clinical vasospasm, cerebral infarction, GOS } \\
\text { at discharge, and mortality }\end{array}$ & $\begin{array}{l}\text { Computer } \\
\text { random }\end{array}$ & Unclear & 6 \\
\hline Sun (11) & 2014 & 76 & 72 & $\begin{array}{l}\text { Intracerebral hemorrhage, vasospasm, } \\
\text { chronic hydrocephalus incidence, tube timing, } \\
\text { infection, and GOS }\end{array}$ & Random & Average of 56.8 & 2 \\
\hline Klimo (14) & 2004 & 81 & 86 & $\begin{array}{l}\text { Anascular spasm, cerebral infarction, and } \\
\text { GOS }\end{array}$ & Random & $18-68$ & 3 \\
\hline Kasuya (15) & 1991 & 92 & 16 & Brain infarction and hydrocephalus & Random & Unclear & Unclear \\
\hline
\end{tabular}

CVS, cerebral vasospasm; GOS, Glasgow Outcome Scale.

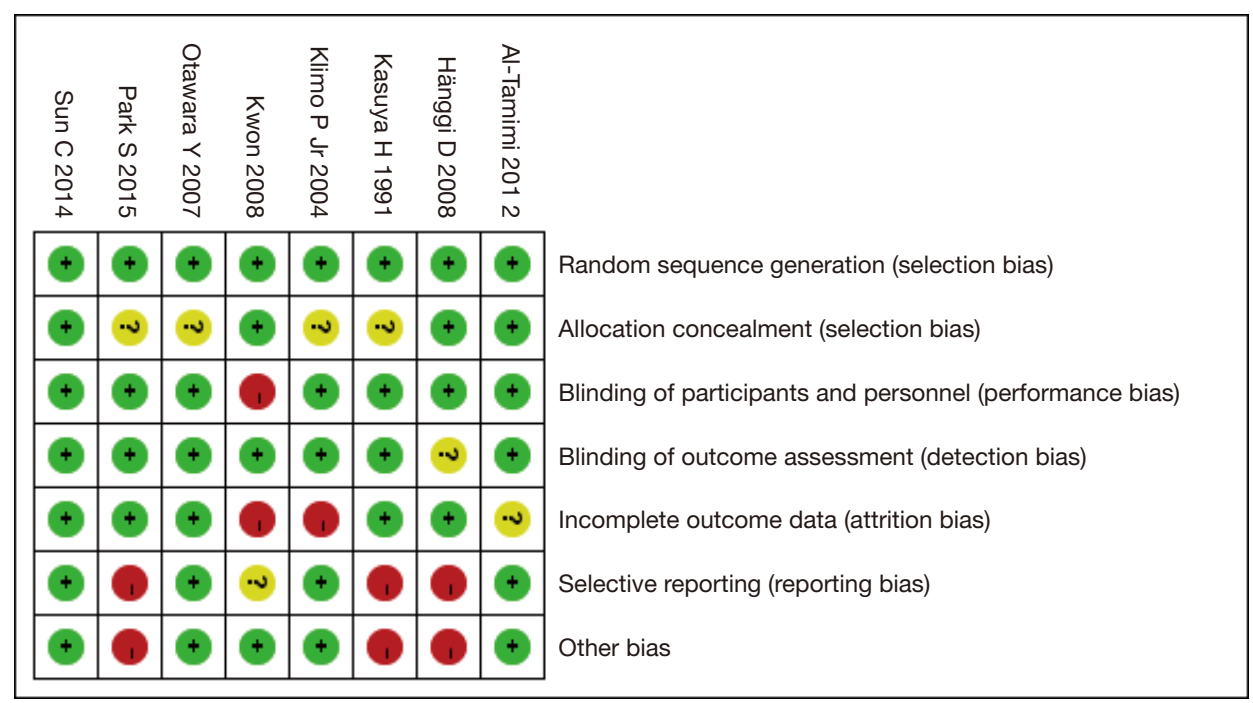

Figure 2 Assessment of bias risk for the included articles.

which indicated significant heterogeneity in the literature; consequently, a random-effects model was used. The metaanalysis results showed OR $=4.76,95 \%$ CI: $(2.11,10.76)$, $\mathrm{Z}=3.75$, and $\mathrm{P}=0.0002$, these results showed a significant difference in the incidence of continuous lumbar CSF drainage between the drainage and conventional groups
$(\mathrm{P}<0.05)$. A forest map is shown in Figure 6. The funnel diagram was symmetrical (Figure 7), with most of the data corresponding to points within the $95 \%$ CI. The nonsafety number was 186 , this suggested that an incidence of cerebral hemorrhage above $5 \mathrm{k}+10=95$ indicated that publication bias. 


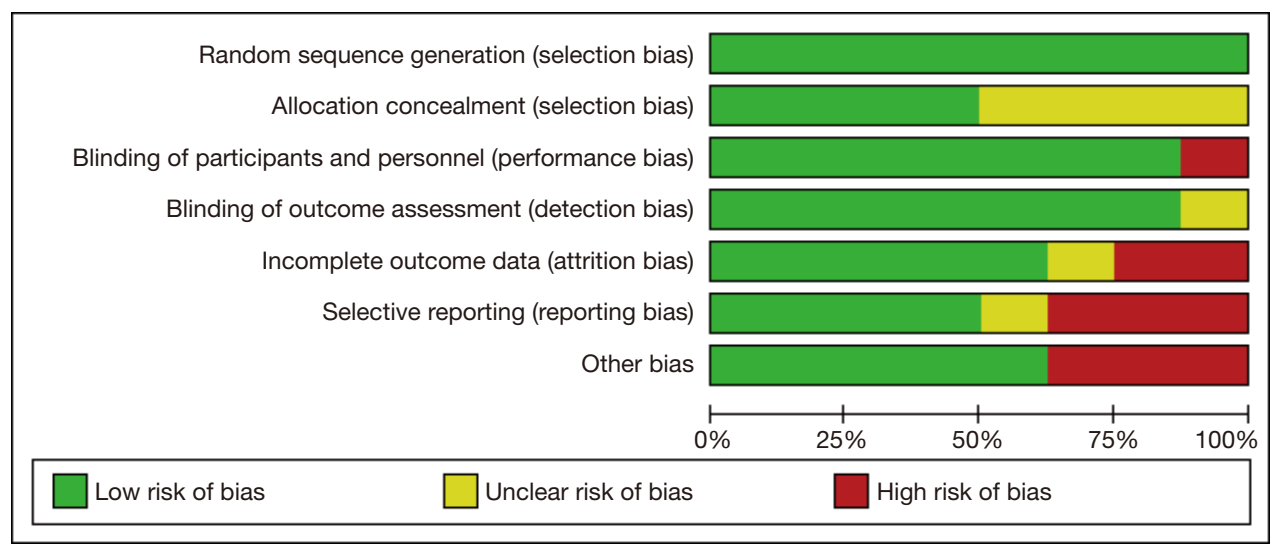

Figure 3 Multiple studies in the articles correspond to the results of risk of bias assessment for the included articles.

\begin{tabular}{|c|c|c|c|c|c|c|c|c|c|c|}
\hline \multirow[b]{2}{*}{ Study or Subqroup } & \multicolumn{2}{|c|}{ Experimental } & \multicolumn{2}{|c|}{ Control } & \multicolumn{2}{|r|}{ Odds Ratio } & \multirow{2}{*}{\multicolumn{4}{|c|}{$\begin{array}{c}\text { Odds Ratio } \\
\mathrm{M}-\mathrm{H}, \text { Random, } 95 \% \mathrm{Cl} \\
\end{array}$}} \\
\hline & Events & Total & Events & Total & Weight & M-H, Random, $95 \% \mathrm{Cl}$ & & & & \\
\hline Al-Tamimi 2012 & 36 & 105 & 7 & 105 & $29.1 \%$ & $7.30[3.07,17.37]$ & & & $\longrightarrow$ & \\
\hline Kasuya H 1991 & 9 & 92 & 2 & 16 & $17.8 \%$ & $0.76[0.15,3.89]$ & & & & \\
\hline Klimo P Jr 2004 & 7 & 81 & 4 & 86 & $22.7 \%$ & $1.94[0.55,6.89]$ & & & & \\
\hline Park S 2015 & 20 & 126 & 11 & 108 & $30.4 \%$ & $1.66[0.76,3.65]$ & & & & \\
\hline Total $(95 \% \mathrm{Cl})$ & & 404 & & 315 & $100.0 \%$ & $2.30[0.90,5.89]$ & & & & \\
\hline Total events & 72 & & 24 & & & & & & & \\
\hline $\begin{array}{l}\text { Heterogeneity: Tau } \\
\text { Test for overall effec }\end{array}$ & $\begin{array}{l}0.59 ; \mathrm{Chi} \\
=1.74\end{array}$ & $\begin{array}{l}=9.17, \\
=0.08\end{array}$ & $d f=3(P$ & $=0.03)$ & $i^{2}=67 \%$ & & 0.001 & 0.1 & 10 & 1000 \\
\hline
\end{tabular}

Figure 4 Forest map comparing the incidence of hydrocephalus in the experimental and control groups.

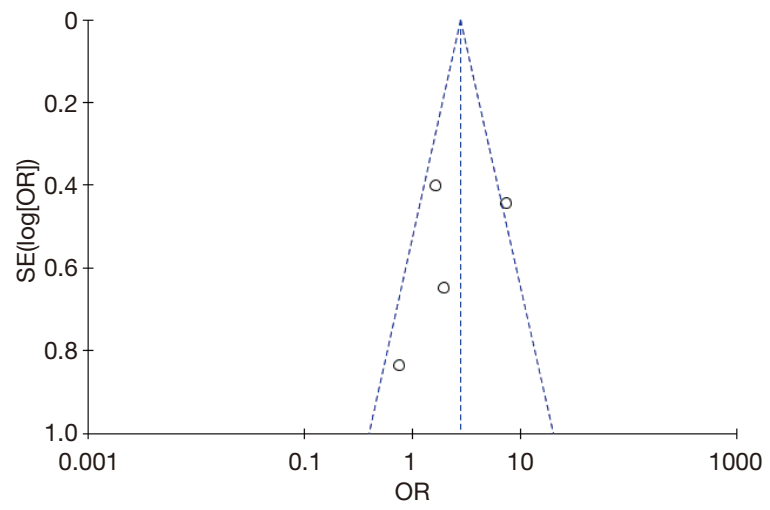

Figure 5 Funnel plot for the incidence of hydrocephalus in the experimental and control groups.

\section{Cerebral infarction}

All 8 studies reported the incidence of cerebral infarction during hospitalization. Cerebral infarction occurred 585 patients in the drainage group and 507 patients in the conventional group. The results of heterogeneity testing showed $\mathrm{I}^{2}=64 \%$ and $\mathrm{P}=0.006$, which indicated significant heterogeneity in the literature; consequently, a random-effects model was used. The meta-analysis results showed $\mathrm{OR}=5.42$, 95\% CI: $(2.71,10.83), Z=4.78$, and $\mathrm{P}<0.00001$, these results showed a significant difference in the incidence of continuous lumbar CSF drainage between the drainage and conventional groups $(\mathrm{P}<0.05)$. A forest map is shown in Figure 8 . The funnel diagram was symmetrical (Figure 9), with most of the data corresponding to points within the $95 \%$ CI. The nonsafety number was 213 , which indicated that at least 213 nonpositive controls were required for to bring hypertension and cerebrovascular disease-related outcomes above $5 \mathrm{k}+10=95$, suggesting that an incidence of cerebral infarction above $5 \mathrm{k}+10=95$ indicated that publication bias.

\section{Late-onset nerve injury}

All 8 studies reported the incidence of delayed nerve injury during hospitalization or follow-up. Delayed nerve injury occurred in 585 patients in the drainage group and 507 patients in the conventional group. The results 


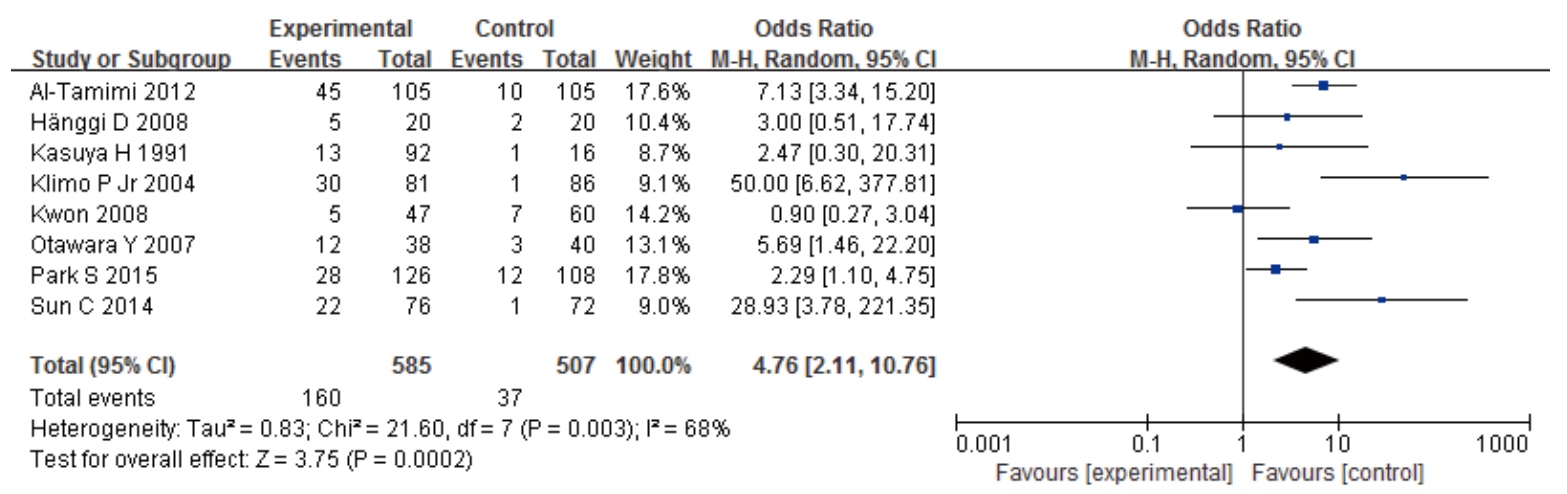

Figure 6 Forest map comparing the incidence of perioperative cerebral hemorrhage in the experimental and control groups.

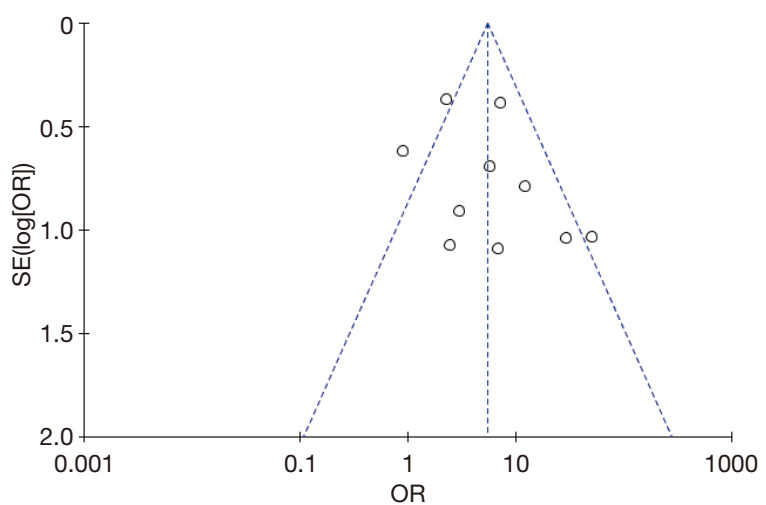

Figure 7 Funnel plot for the incidence of perioperative cerebral hemorrhage in the experimental and control groups.

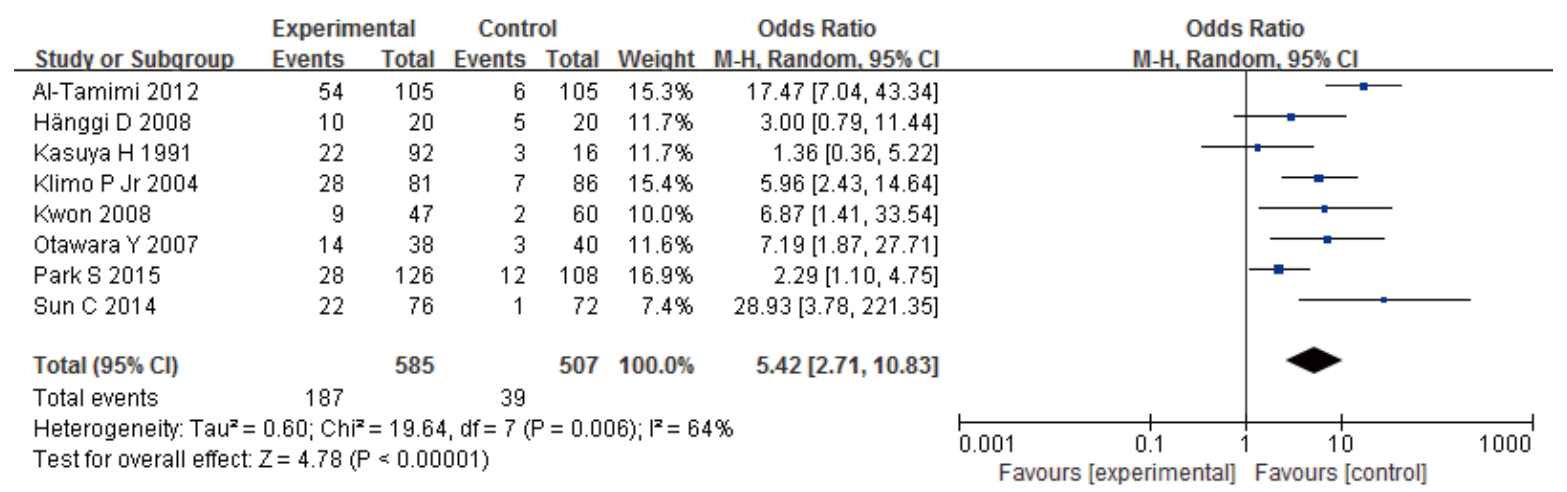

Figure 8 Forest map comparing the incidence of perioperative cerebral infarction in the experimental and control groups.

of heterogeneity testing showed $\mathrm{I}^{2}=0 \%$ and $\mathrm{P}=0.58$, which indicated obvious heterogeneity in the literature; consequently, a fixed-effects model was used. The results of the meta-analysis showed OR =3.62, 95\% CI: $(2.10,6.22)$, $\mathrm{Z}=4.65$, and $\mathrm{P}<0.00001$; therefore, significant differences in the incidence of delayed nerve injury caused by persistent CSF drainage existed between the two groups $(\mathrm{P}<0.05)$. A forest map is shown in Figure 10. The funnel diagram was symmetrical (Figure 11), with most of the data corresponding to the $95 \%$ CI points. The non-safety number was 92 , 
which indicated that at least 92 non-positive controls were required to produce hypertension and cerebrovascular disease-related outcomes above $5 \mathrm{k}+10=95$, suggesting that an incidence of delayed nerve injury above $5 \mathrm{k}+10=95$ indicated that publication bias.

\section{Infection}

All 8 studies reported the incidence of infection during hospitalization or follow-up. Infection occurred in 585 patients in the drainage group and 507 patients in the conventional group. The results of heterogeneity testing showed $\mathrm{I}^{2}=15 \%$ and $\mathrm{P}=0.31$, which indicated obvious heterogeneity in the literature; consequently, a fixedeffects model was used. The meta-analysis results showed $\mathrm{OR}=4.12,95 \% \mathrm{CI}:(2.75,6.18), \mathrm{Z}=6.83$, and $\mathrm{P}<0.00001$. Therefore, there was a significant difference in the incidence of perioperative infection between the two groups

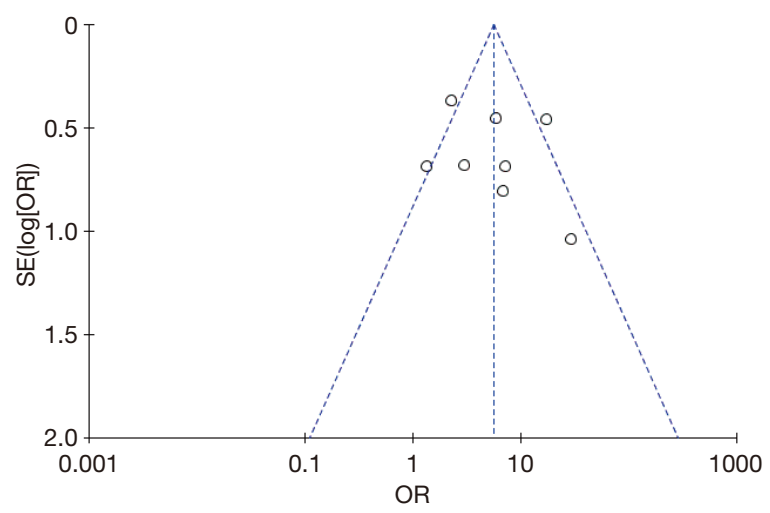

Figure 9 Funnel plot for the incidence of perioperative cerebral infarction in the experimental and control groups. of patients $(\mathrm{P}<0.05)$. A forest map is shown in Figure 12 . The funnel diagram was symmetric (Figure 13), with most of the data corresponding to points within the $95 \%$ CI. The non-safety number was 148 , which indicated that at least 148 non-positive controls were needed to bring hypertension and cerebrovascular disease-related outcomes above $5 \mathrm{k}+10=95$, suggesting that an incidence of delayed nerve injury above $5 \mathrm{k}+10=95$ indicated that publication bias.

\section{Glasgow Outcome Scale (GOS)}

Five studies reported patients' Glasgow Prognostic Score (GPS) during hospitalization or follow-up. The GOS was reported for 345 patients in the drainage group and 287 patients in the conventional group. Heterogeneity test results showed $\mathrm{I}^{2}=0 \%$ and $\mathrm{P}=0.76$, which indicated significant heterogeneity in the literature; consequently, a fixed-effects model was used. The results of the meta-analysis showed

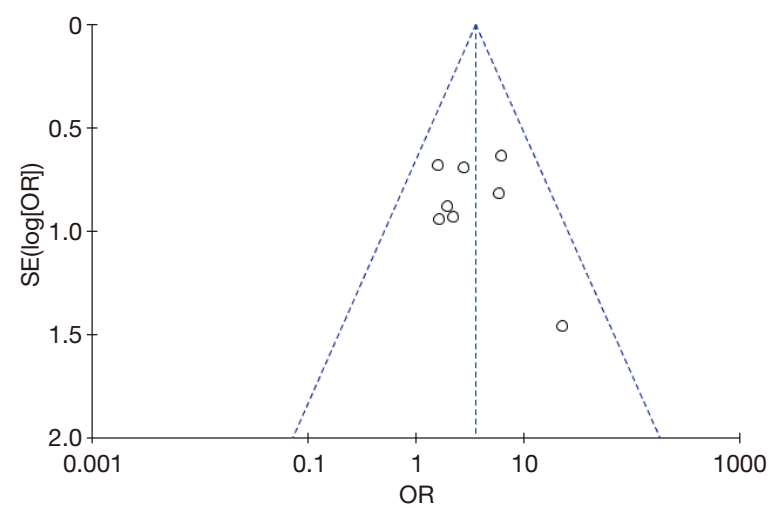

Figure 11 Funnel plot for the incidence of delayed nerve injury in the experimental and control groups.

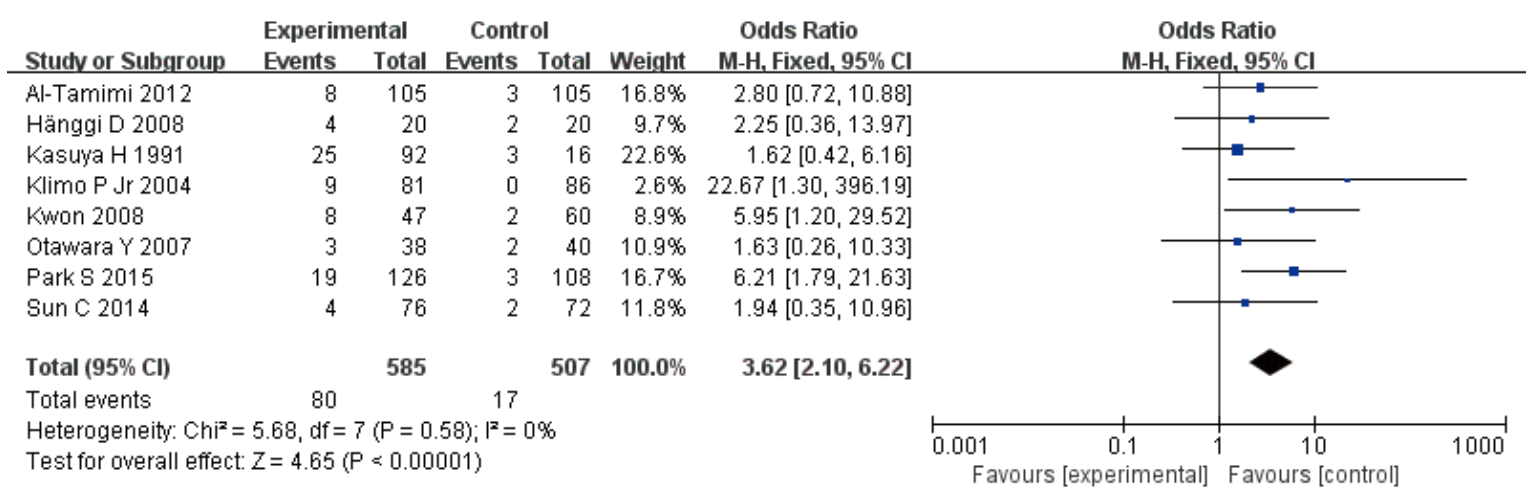

Figure 10 Forest map comparing the incidence of delayed perioperative nerve injury in the experimental and control groups. 


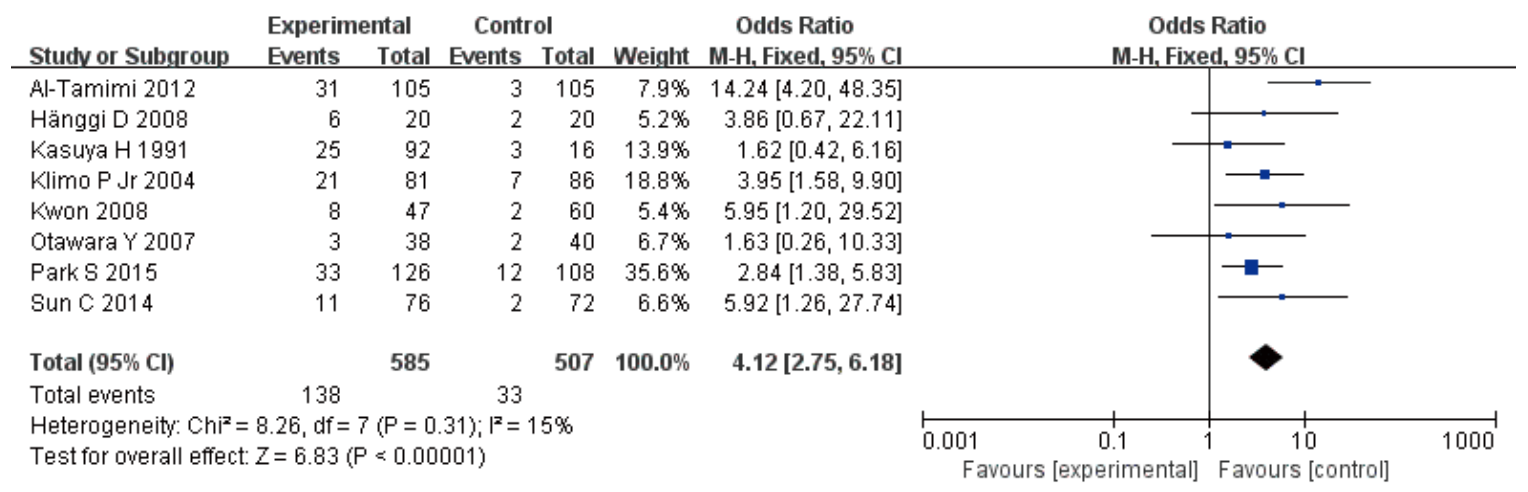

Figure 12 Forest map comparing the perioperative incidence of perforation infection the experimental and control groups.

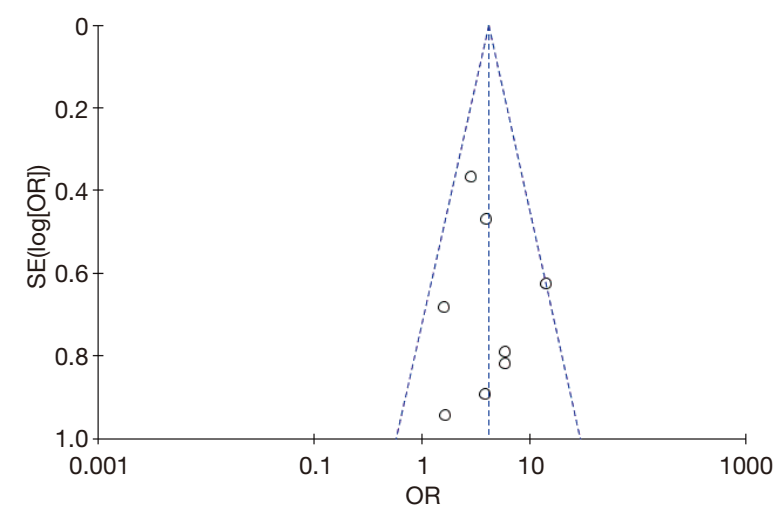

Figure 13 Funnel plot for the incidence of perioperative infection in the experimental and control groups.

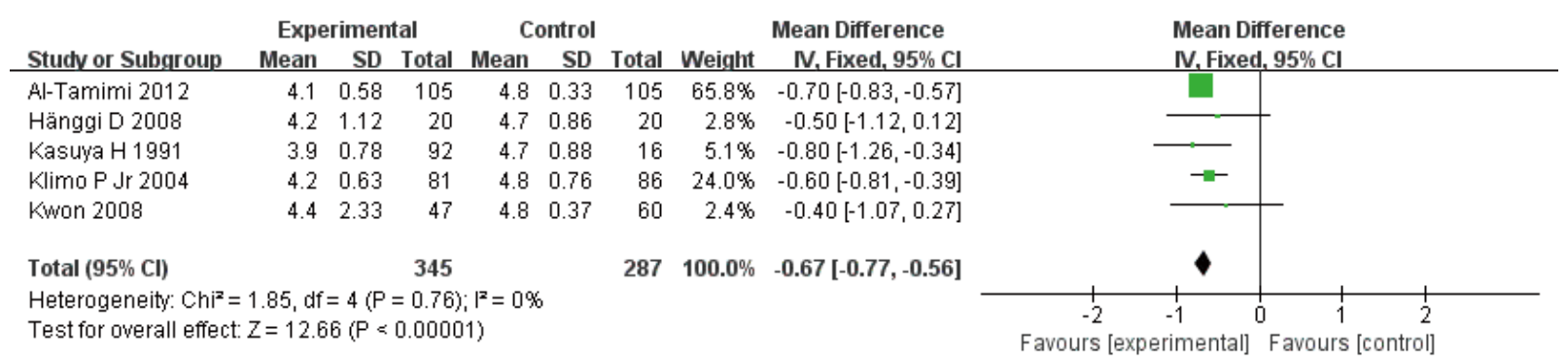

Figure 14 Forest plot comparing GPS during the perioperative period of keyhole surgery in the experimental and control groups. GPS, Glasgow Prognostic Score.

mean ratio $(M R)=-0.67,95 \% \mathrm{CI}:(-0.77,-0.56), \mathrm{Z}=12.66$, and $\mathrm{P}<0.00001$; therefore, significant differences in GPS existed between the two groups $(\mathrm{P}<0.05)$. A forest map is shown in Figure 14. The funnel diagram was symmetrical (Figure 15), with most of the data corresponding to points within the $95 \%$ CI. The non-safety number was 32, which indicated that at least 32 non-positive controls were required to produce hypertension and cerebrovascular disease-related outcomes above $5 \mathrm{k}+10=95$, suggesting that an incidence of GOS above $5 \mathrm{k}+10=95$ indicated that publication bias.

\section{Discussion}

The effect of CSF drainage of lumbar cistern on the 


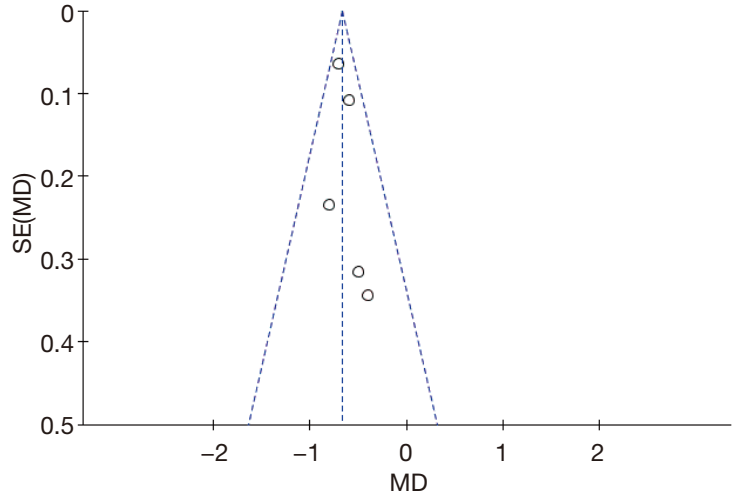

Figure 15 Funnel plot for the perioperative GPS in the experimental and control groups. GPS, Glasgow Prognostic Score.

prognosis of patients, especially on the mortality rate, remains to be studied. Therefore, according to the Cochrane systematic evaluation method, this study searched RCTs on improving the prognosis of patients with CSF drainage of lumbar cistern to verify the effectiveness and safety of drainage. This meta-analysis included 8 RCTs of continuous lumbar pool drainage (8-15). Of the 8 selected studies, seven reported the age, sex, prognostic indicators, and follow-up time of patients, with no statistically significant differences between the drainage and control groups $(\mathrm{P}>0.05)$. All 8 studies randomly divided patients into drainage and control groups, but none of them reported blindness, and none of the articles reported the number of deaths during the study. The limitations of the interventions in this study indicated that measurement deviations could exist between drainage and control patients. To further the research reliability and reference ability, research methods and designs should be improved in the future.

Continuous lumbar pool CSF drainage is routinely used to treat aSAH, and can reduce mortality and complications among patients with this condition $(16,17)$. Locking neurosurgery is suitable for patients with intracranial aneurysm, hypertension, cerebral hemorrhage, and other diseases. Continuous lumbar pool CSF drainage significantly influences the perioperative period of locking surgery $(18,19)$. The preliminary results of our metaanalysis of 8 original RCTs suggest that continuous drainage of CSF can reduce a number of perioperative complications in patients undergoing locking surgery, including hydrocephalus, cerebral hemorrhage, cerebral infarction, and delayed nerve damage, while improving the GPS; thus, it has a critical impact on patient prognosis. Early craniotomy of aneurysm neck clipping or endovascular intervention after onset can well control rebleeding. There are many treatments for CVS, including nimodipine, nicardipine, fasudil, endothelin receptor antagonist, magnesium, statins, and endovascular balloon angioplasty. However, the control of CVS is still an important part in the prevention of high mortality of aSAH (20). To date, there have been few reports on the impact of continuous drainage of CSF on the perioperative period of perforation surgery, intracranial or puncture site infection, low intracranial pressure, and cerebral hernia nerve root damage or stimulation. These indicators need to be closely observed in future studies $(21,22)$, such as intracranial or puncture site infection, low cranial pressure, hernia nerve root damage or irritation. Some scholars believe that continuous lumbar pool drainage can improve clinical symptoms but does not reduce permanent nerve damage caused by CVS (23). Decline of intracranial pressure increases the possibility of aneurysmal re-rupture and bleeding, which suggests that the advantages and disadvantages should be considered before continuous drainage (24). Even with the blinding of patients and researchers, the use of both invasive and noninvasive procedures could lead to a high risk of incomplete implementation of the blinding method, which may produce information bias and thus affect the reliability of the meta-analysis.

\section{Conclusions}

Our results confirm that continuous lumbar pool drainage can reduce the incidence of some complications in the perioperative period of keyhole surgery, including cerebral hemorrhage, hydrocephalus, cerebral infarction, infection, and delayed nerve injury, while increasing the GPS. However, current studies do not provide sufficient evidence for the clinical promotion and application of continuous lumbar pool drainage, and more high-quality, large-sample, and multicenter RCTs should be conducted to obtain more reliable evidence.

\section{Acknowledgments}

Funding: None.

\section{Footnote}

Reporting Checklist: The authors have completed the PRISMA reporting checklist. Available at https://dx.doi. 
org/10.21037/apm-21-2728

Conflicts of Interest: All authors have completed the ICMJE uniform disclosure form (available at https://dx.doi. org/10.21037/apm-21-2728). The authors have no conflicts of interest to declare.

Ethical Statement: The authors are accountable for all aspects of the work in ensuring that questions related to the accuracy or integrity of any part of the work are appropriately investigated and resolved.

Open Access Statement: This is an Open Access article distributed in accordance with the Creative Commons Attribution-NonCommercial-NoDerivs 4.0 International License (CC BY-NC-ND 4.0), which permits the noncommercial replication and distribution of the article with the strict proviso that no changes or edits are made and the original work is properly cited (including links to both the formal publication through the relevant DOI and the license). See: https://creativecommons.org/licenses/by-nc-nd/4.0/.

\section{References}

1. Hussein $M$, Abdellatif M. Continuous lumbar drainage for the prevention and management of perioperative cerebrospinal fluid leakage. Asian J Neurosurg 2019;14:473-8.

2. Kırık A, Yaşar S. The efficacy of continuous lumbar drainage in post-traumatic cerebrospinal fluid fistulas. Ulus Travma Acil Cerrahi Derg 2020;26:784-8.

3. Ringel B, Carmel-Neiderman NN, Peri A, et al. Continuous lumbar drainage and the postoperative complication rate of open anterior skull base surgery. Laryngoscope 2018;128:2702-6.

4. Bakhshi SK, Suhail N, Mitha R, et al. Lumbar drain for temporary cerebrospinal fluid diversion: factors related to the risks of complications at a university hospital. World Neurosurg 2020;143:e193-8.

5. Takase K, Yoshida T, Nakamura T, et al. Cryptococcal meningitis in a patient with breast cancer receiving everolimus: a case of successful treatment with continuous cerebrospinal fluid drainage. Brain Nerve 2018;70:1301-5.

6. Zhang J, Hua W, Zhang X, et al. Pure endoscopic surgery via subtemporal extradural keyhole approach for middle cranial fossa tumors. World Neurosurg 2019;130:e487-97.

7. Kotwica Z, Saracen A, Kasprzak P. Keyhole surgery of pineal area tumors - personal experience in 22 patients.
Transl Neurosci 2017;8:207-10.

8. Al-Tamimi YZ, Bhargava D, Feltbower RG, et al. Lumbar drainage of cerebrospinal fluid after aneurysmal subarachnoid hemorrhage: a prospective, randomized, controlled trial (LUMAS). Stroke 2012;43:677-82.

9. Kwon OY, Kim YJ, Kim YJ, et al. The utility and benefits of external lumbar CSF drainage after endovascular coiling on aneurysmal subarachnoid hemorrhage. J Korean Neurosurg Soc 2008;43:281-7.

10. Park S, Yang N, Seo E. The effectiveness of lumbar cerebrospinal fluid drainage to reduce the cerebral vasospasm after surgical clipping for aneurysmal subarachnoid hemorrhage. J Korean Neurosurg Soc 2015;57:167-73.

11. Sun C, Du H, Yin L, et al. Choice for the removal of bloody cerebrospinal fluid in postcoiling aneurysmal subarachnoid hemorrhage: external ventricular drainage or lumbar drainage? Turk Neurosurg 2014;24:737-44.

12. Hänggi $\mathrm{D}$, Liersch J, Turowski B, et al. The effect of lumboventricular lavage and simultaneous lowfrequency head-motion therapy after severe subarachnoid hemorrhage: results of a single center prospective Phase II trial. J Neurosurg 2008;108:1192-9.

13. Otawara Y, Ogasawara K, Kubo Y, et al. Effect of continuous cisternal cerebrospinal fluid drainage for patients with thin subarachnoid hemorrhage. Vasc Health Risk Manag 2007;3:401-4.

14. Klimo P Jr, Kestle JR, MacDonald JD, et al. Marked reduction of cerebral vasospasm with lumbar drainage of cerebrospinal fluid after subarachnoid hemorrhage. J Neurosurg 2004;100:215-24.

15. Kasuya H, Shimizu T, Kagawa M. The effect of continuous drainage of cerebrospinal fluid in patients with subarachnoid hemorrhage: a retrospective analysis of 108 patients. Neurosurgery 1991;28:56-9.

16. Filis AK, Aghayev K, Vrionis FD. Cerebrospinal fluid and hydrocephalus: physiology, diagnosis, and treatment. Cancer Control 2017;24:6-8.

17. Girard S, Fenneteau O, Mestrallet F, et al. Recommendations for cerebrospinal fluid examination in acute leukemia. Ann Biol Clin (Paris) 2017;75:503-12.

18. Rupa V, Joy N. Management of coexistent sinonasal pathology in patients undergoing endoscopic cerebrospinal fluid rhinorrhea repair. Braz J Otorhinolaryngol 2020. [Epub ahead of print]. doi: 10.1016/j.bjorl.2020.11.010.

19. Kobayashi K, Ando K, Ito K, et al. Efficacy of intraoperative lumbar subarachnoid drainage for prevention of cerebrospinal fluid leak after spinal cord 
tumor resection. J Orthop Sci 2018;23:266-72.

20. Fang Y, Shao Y, Lu J, et al. The effectiveness of lumbar cerebrospinal fluid drainage in aneurysmal subarachnoid hemorrhage with different bleeding amounts. Neurosurg Rev 2020;43:739-47.

21. Qian C, Yu X, Chen J, et al. Effect of the drainage of cerebrospinal fluid in patients with aneurismal subarachnoid hemorrhage: a meta-analysis. Medicine (Baltimore) 2016;95:e5140.

22. Chen C, Xu P, Xu T, et al. Influence of cerebrospinal fluid drainage and other variables on the plasma vancomycin trough levels in postoperative neurosurgical patients. Br J
Neurosurg 2021;35:133-8.

23. Findlay JM, Kassell NF, Weir BK, et al. A randomized trial of intraoperative, intracisternal tissue plasminogen activator for the prevention of vasospasm. Neurosurgery 1995;37:168-76; discussion 177-8.

24. Ruijs AC, Dirven CM, Algra A, et al. The risk of rebleeding after external lumbar drainage in patients with untreated ruptured cerebral aneurysms. Acta Neurochir (Wien) 2005;147:1157-61; discussion 1161-2 .

(English Language Editor: J. Chapnick)
Cite this article as: Feng J, Liu Q, Cao D, Liu F. Systematic review and meta-analysis of the effect of continuous cerebrospinal fluid drainage on keyhole surgery during the perioperative period. Ann Palliat Med 2021;10(10):1112911140. doi: 10.21037/apm-21-2728 